\title{
Cost Effectiveness of Antenatal Lifestyle Interventions for Preventing Gestational Diabetes and Hypertensive Disease in Pregnancy
}

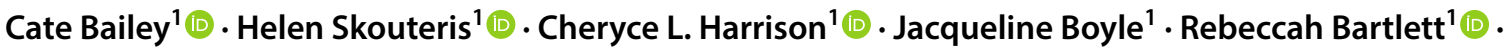

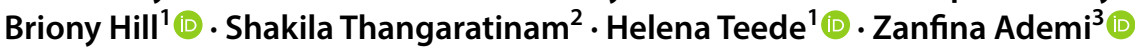

Published online: 5 February 2020

(c) The Author(s) 2020

\begin{abstract}
Background Lifestyle interventions (diet, physical activity and/or behavioural) to optimise gestational weight gain can prevent adverse maternal outcomes such as gestational diabetes, pre-eclampsia and caesarean section.

Objective We aimed to model the cost effectiveness of lifestyle interventions during pregnancy on reducing adverse maternal outcomes.

Methods Decision tree modelling was used to determine the cost effectiveness of lifestyle interventions compared with usual care on preventing cases of gestational diabetes and hypertensive disease in pregnancy. Participants were pregnant women receiving routine antenatal care in secondary and tertiary care hospitals. The main outcome measures were cases of gestational diabetes and/or hypertensive disease in pregnancy prevented, costs, and incremental cost-effectiveness ratios. Analysis was conducted from the perspective of the Australian healthcare system, with a time horizon of early pregnancy to discharge after birth.

Results Women in the intervention group were $2.25 \%$ less likely to have gestational diabetes and/or hypertensive disease in pregnancy (9.53\%) compared with the control group (11.78\%). Intervention costs were Australian dollars (AUD) 228 per person. Costs were AUD33 per person higher in the intervention group (AUD8281) than the control group (AUD8248). The incremental cost-effectiveness ratio was AUD1470 per case prevented. Sensitivity analysis showed that base-case results were robust. In the probabilistic sensitivity analysis, $44.8 \%$ of data points fell within the north-east quadrant, and $52.2 \%$ in the south-east quadrant (cost saving), with a 95\% confidence interval ranging from AUD - 50,018 to 32,779 per case prevented. Conclusions While there is no formally accepted cost-effectiveness threshold for willingness-to-pay to prevent an adverse maternal event, the cost per person receiving a lifestyle intervention compared with controls was close to neutral, and therefore likely to be cost effective. Exploration of the cost effectiveness of different lifestyle delivery modes across various models of antenatal care is now required. Future cost-effectiveness studies should investigate longer time horizons, qualityadjusted life-years and productivity loss.
\end{abstract}

Trial Registration Not applicable.

Helena Teede and Zanfina Ademi shared joint senior authorship.

Electronic supplementary material The online version of this article (https://doi.org/10.1007/s41669-020-00197-9) contains supplementary material, which is available to authorized users.

Zanfina Ademi

zanfina.ademi@monash.edu

1 Monash Centre for Health Research and Implementation, School of Public Health and Preventive Medicine, Monash University, Melbourne, VIC, Australia
2 Women's Health Research Unit, Barts and the London, School of Medicine and Dentistry, Queen Mary University of London, London, UK

3 School of Public Health and Preventive Medicine, Monash University, Melbourne, VIC, Australia 


\section{Key Points for Decision Makers}

Lifestyle interventions may be cost effective (incremental cost-effectiveness ratio $=$ Australian dollars [AUD] 1470), with sensitivity analysis results suggesting that interventions could be cost saving.

There is potential for greater economic benefits when long-term maternal and child health benefits are incorporated into the economic model.

Future research could explore various lifestyle intervention delivery modes and different models of antenatal care, and could incorporate economic impact from a societal perspective and over a longer time-horizon.

\section{Introduction}

Worldwide, obesogenic environments are adversely affecting women's health [1]. Many women enter pregnancy at a higher than recommended weight, and approximately $45 \%$ of women gain weight in pregnancy in excess of international guidelines [2-4]. Excessive gestational weight gain is a risk factor for gestational diabetes and pre-eclampsia, which are common antenatal conditions with serious adverse maternal and infant outcomes over the short and longer term [5-8]. Recent Australian figures suggest that one in seven women will develop gestational diabetes during pregnancy, and that women with gestational diabetes are more likely to be diagnosed with hypertensive disease in pregnancy [9]. These conditions have a large associated cost burden on health care systems. In the United States (US), gestational diabetes is associated with maternal healthcare costs of US $\$ 1.3$ billion [10], and pre-eclampsia with healthcare costs of US $\$ 1.03$ billion for mothers and US $\$ 1.15$ billion for infants [11]. Reducing the incidence of gestational diabetes and pre-eclampsia by implementing lifestyle interventions to limit excessive gestational weight gain could generate major cost savings for health systems.

Evidence synthesis has shown that antenatal diet and physical activity lifestyle interventions for women have been successful in limiting excessive gestational weight gain $[12,13]$. However, the effect of lifestyle interventions on reducing adverse maternal events has been difficult to measure at an individual trial level due to sample size limitations and insufficient power. To address this issue, the International Weight Management in Pregnancy Collaborative Group (iWIP) collected data from all available trials and used meta-analysis to explore intervention effects on a range of prioritised maternal and infant outcomes [14, 15]. The analyses now provide level 1 evidence of the significant benefits of lifestyle intervention on specific pregnancy outcomes, including gestational diabetes, pre-eclampsia and hypertensive disorders of pregnancy, and caesarean section rates. (Note that in the iWIP meta-analysis, papers classified as 'physical activity' were broadly defined, and may have included specific physical activity classes or a behavioural intervention to increase movement.)

While understanding of the effectiveness of lifestyle interventions in pregnancy has progressed, the cost effectiveness of lifestyle interventions is still unclear. Our systematic review of published cost-effectiveness studies demonstrated that only a limited number of economic analyses of lifestyle interventions were conducted alongside individual clinical trials, and the available cost-effectiveness analyses were largely inconclusive [16]. Inadequate power to detect clinical impact in individual studies, over and above optimising gestational weight gain, has limited the usefulness of published cost-effectiveness analyses. In the context of recently demonstrated efficacy of lifestyle interventions through metaanalysis [14], cost-effectiveness modelling is now required to inform clinical guidelines and policy for implementing lifestyle interventions at a population level. The aim of the current study was to model the cost effectiveness of an effective lifestyle intervention during pregnancy, compared with usual care, on reducing adverse maternal outcomes.

\section{Methods}

\subsection{Overview of Approach and Model}

We conducted a model-based cost-effectiveness analysis comparing diet and physical activity interventions (aiming to limit gestational weight gain and/or reduce cases of gestational diabetes) with usual care. The incremental cost-effectiveness ratio (ICER) was expressed as cost per case prevented. Cases included gestational diabetes and/or hypertensive disease in pregnancy (including pre-eclampsia and pregnancy-induced hypertension). A decision tree model (see Fig. 1) was developed for four health states: (1) develop gestational diabetes; (2) develop hypertensive disease in pregnancy; (3) develop gestational diabetes and hypertensive disease in pregnancy; or (4) do not develop gestational diabetes or hypertensive disease in pregnancy. In each health state, data were modelled for aspect of delivery: (1) caesarean section; (2) induction of labour with vaginal birth; (3) induction of labour with caesarean section; or (4) neither induction nor caesarean section. The time horizon was between early pregnancy and the discharge of mother and infant from hospital after birth; hence, it was not necessary to use discounting. Data were analysed from the perspective 


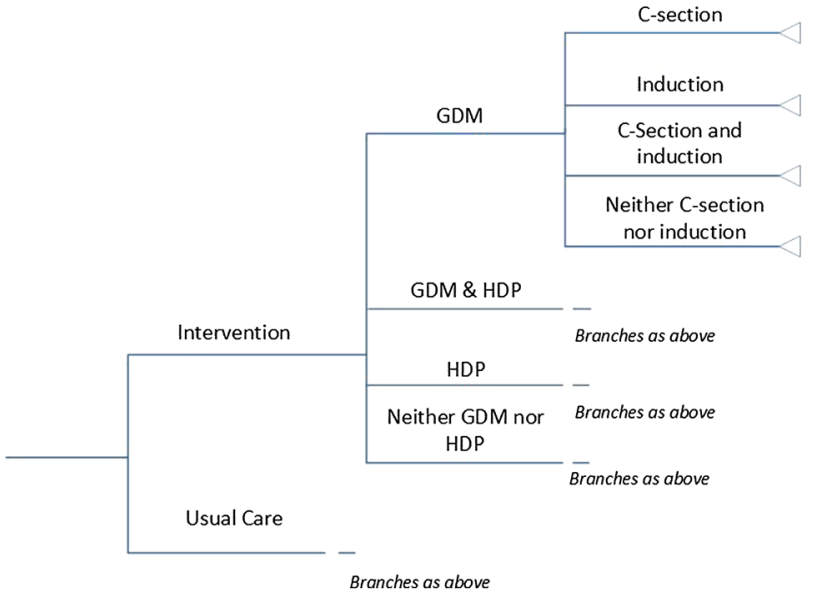

Fig. 1 Decision tree model for intervention versus usual care, health state, and type of delivery. GDM gestational diabetes mellitus, HDP hypertensive disease in pregnancy, $C$-section caesarean section

of the public healthcare system in Australia as any savings from this intervention would have highest benefit for the healthcare funder. We only included health states where there were data from the Monash Health dataset and iWIP meta-analysis comparing intervention and control groups, which then enabled us to model cost effectiveness. Thus, health states such as miscarriage and maternal death were not included in the model.

\subsection{Population}

Inputs for the usual care group in the model were derived from a de-identified Monash Health routine maternity care dataset capturing gestational diabetes and/or hypertensive disease in pregnancy and mode of delivery [17]. Monash Health is one of Australia's largest public health services, providing universal free and accessible care for over 10,000 births per year across multiple hospital sites for a multicultural population, covering urban and regional areas [18]. Antenatal care at Monash Health was largely provided in secondary and tertiary care hospitals. Data were collected between 2009 and 2013 for all singleton pregnancies resulting in births of $>20$ weeks' gestation, and can be considered to be a representative sample of the general population for which the intervention modelled in this paper can apply. The demographics of the region are representative of much of the Australian population, with significant ethnic diversity and variation in socioeconomic status. Healthcare is provided in a universally accessible public health system and the population is generally representative of the vast majority of women who attend public health services. Australia has a multi-payer health system and some women use private insurance for obstetric care. Those women accessing private obstetric care are generally more educated and affluent and more likely to have been Australian born. They are not captured in this dataset.

In the Monash Health dataset, body mass index (BMI) data were categorised as per World Health Organization recommendations [19]. The dataset contained 38,052 cases, of which 20,341 women $(53.5 \%)$ had a BMI $<25 \mathrm{~kg} / \mathrm{m}^{2}$, 10,112 women (26.6\%) had a BMI over 25 to up to $30 \mathrm{~kg} / \mathrm{m}^{2}$, and 7599 women $(20.0 \%)$ had a BMI of $30 \mathrm{~kg} / \mathrm{m}^{2}$ plus. The mean age of health service users was 29.7 years (standard deviation 5.43), and users were mainly English speaking $(n=25,948 ; 70.1 \%)$, multiparous $(n=21,764 ; 55.7 \%)$ and non-smoking $(n=31,818 ; 82.9 \%)$. In the dataset, $7.5 \%$ of health service users were diagnosed with gestational diabetes, $3.8 \%$ with hypertensive disease in pregnancy, and $0.5 \%$ with both, while $27.8 \%$ of all health service users were delivered via caesarean section and $21.9 \%$ were induced. Monash Health applied national guidelines for the diagnosis of gestational diabetes based on large interventional trials [20,21]. For mode of delivery, percentages of cases with caesarean sections, induction with vaginal birth, induction with caesarean section, or vaginal birth only were extracted for each health state (gestational diabetes, hypertensive disease in pregnancy, both, none of the above) and are presented in Table 1. No maternal deaths occurred over 4 years of follow up in the Monash Health dataset, and, as such, we have not included maternal death in the model.

\subsection{Usual Care}

At the time of data collection, usual care in this group consisted of ad hoc and limited gestational weight monitoring (i.e. not part of routine care), written information on diet
Table 1 Mode of birth by health state: percentages of cases of caesarean section, induction with vaginal birth, induction with caesarean birth, and vaginal birth only for each health state

\begin{tabular}{lllcc}
\hline Full sample & Caesarean section & $\begin{array}{l}\text { Induction with } \\
\text { vaginal birth }\end{array}$ & $\begin{array}{l}\text { Induction with cae- } \\
\text { sarean section }\end{array}$ & $\begin{array}{l}\text { Vaginal } \\
\text { birth only }\end{array}$ \\
\hline Gestational diabetes & 26.32 & 21.98 & 8.64 & 43.06 \\
$\begin{array}{l}\text { Hypertensive disease in } \\
\text { pregnancy }\end{array}$ & 24.32 & 43.04 & 18.72 & 13.92 \\
Both & 31.22 & 33.33 & 22.75 & 12.70 \\
None of the above & 21.54 & 14.34 & 4.76 & 59.36 \\
\hline
\end{tabular}


and food hygiene, and safety per national dietary guidelines for pregnancy. Women received no lifestyle intervention or dietary advice during pregnancy on the prevention of excess gestational weight gain.

\subsection{Lifestyle Intervention Effects}

The effect of lifestyle intervention compared with usual care was obtained from a meta-analysis of lifestyle interventions during pregnancy authored by the iWIP [14]. In the metaanalysis, 59 studies contained information on gestational diabetes (16,185 women), 45 studies contained information on hypertensive disease in pregnancy (14,849 women) and 66 studies contained information on caesarean section $(18,041$ women). Adjusted odds ratios (ORs) as presented in the iWIP meta-analysis for gestational diabetes were 0.76 (95\% confidence interval [CI] 0.65-0.89); hypertensive disease in pregnancy, 0.85 (95\% CI 0.71-1.00); and caesarean section, 0.89 (95\% CI 0.83-0.96).

Adjusted ORs as presented in the iWIP meta-analysis [14] were converted to relative risks (RRs) using the following formula: $\mathrm{RR}=\mathrm{OR} /(1-p+(p \times \mathrm{OR}))$, where $p$ represents the risk in the control group [22]. RR (risk ratio) is used here as it the ratio of two probabilities, rather than the OR, which, as implied by its name, is the ratio of two odds. We used the RR because the OR may exaggerate the risk when an outcome is common, [23]. To derive the intervention effect, we multiplied the RRs from the iWIP meta-analysis by the proportion of cases in each health state, as derived from the Monash Health dataset (usual care). Risk ratios with 95\% CIs are presented in Table 2. Risk ratios for induction were set to 1 as there was no information in the iWIP metaanalysis to attach to this variable.

\subsection{Lifestyle Intervention Costs}

Intervention costs were estimated from a lifestyle intervention evaluated in a randomised controlled trial of 228 pregnant women at high risk of developing gestational diabetes, delivered embedded with routine care at Monash Health [24, 25]. Inclusion criteria for the trial were singleton pregnancies, entrance to the study in early pregnancy (12-15 weeks gestation), no pre-existing diabetes (type 1 or 2 diabetes mellitus) and at high risk of developing gestational diabetes identified using a validated gestational diabetes risk screening tool [26]. Women were randomised to either an intervention or a control group, as previously described [24]. Intervention costs were estimated for four behavioural intervention sessions of 30 min' duration plus administration and contact costs, as shown in electronic supplementary Table S1. The intervention was estimated to cost Australian dollars (AUD) 228 per person, at 2019 prices. As outlined above, the trial was powered to detect differences between
Table 2 Key input parameters, including parameter variation and distribution

\begin{tabular}{lllll}
\hline Risk ratios & Base & \multicolumn{2}{l}{$95 \%$ CI } & Distribution \\
\cline { 2 - 4 } & & Lower & Upper & \\
\hline GDM risk ratio & 0.789 & 0.681 & 0.903 & Normal \\
HDP risk ratio & 0.863 & 0.727 & 1.000 & Normal \\
GDM and HDP risk ratio & 0.789 & 0.681 & 0.903 & Normal \\
C-section risk ratio & 0.920 & 0.871 & 0.971 & Normal \\
Induction & 1 & & & \\
Costs AUD & Base & $-30 \%$ & $+30 \%$ & \\
Antenatal GDM costs & 1055 & 738 & 1371 & Gamma \\
Antenatal GDM and HDP & 2781 & 1947 & 3616 & Gamma \\
$\quad$ costs & & & & \\
Antenatal HDP costs & 1923 & 1346 & 2500 & Gamma \\
Intervention costs & 228 & 160 & 296 & Gamma \\
Vaginal birth costs & 5812 & 4068 & 7555 & Gamma \\
C-section costs & 11,416 & 7992 & 14,841 & Gamma \\
Induction and vaginal birth & 7846 & 5492 & 10,200 & Gamma \\
Induction only & 2034 & 1424 & 2645 & Gamma \\
\hline
\end{tabular}

AUD Australian dollars, $C I$ confidence interval, GDM gestational diabetes mellitus, $H D P$ hypertensive disease in pregnancy, $C$-section caesarean section

groups in gestational weight gain, but was not powered to detect differences between groups for health states including gestational diabetes, hypertensive disease in pregnancy or birth/delivery mode. Hence, we modelled the intervention effect for gestational diabetes and hypertensive disease in pregnancy from the iWIP meta-analysis [14]. Data from this study were included in the iWIP dataset.

\subsection{Public Health Care Costs}

\subsubsection{Patient Pathways}

Health service use for pregnancy-related conditions was determined by developing separate patient pathways for women with (1) gestational diabetes; (2) hypertensive disease in pregnancy; or (3) both, to determine an approximation of the type and quantity of services likely to have been accessed by health service users. Patient pathways were developed through the use of clinical guidelines, Monash Health pathways and policies [27-29], relevant publications [7, 30, 31] and discussion with Monash Health clinicians (Monash Health Gynaecologist/Obstetrician $[\mathrm{JB}]$, Endocrinologist $[\mathrm{HT}]$ and Midwife [RB]). The average costs for these pathways were calculated for the cost year of 2019. The cost of antenatal gestational diabetes was estimated as AUD1055, antenatal hypertensive disease in pregnancy was estimated as AUD1723, and the cost of having both conditions was estimated as AUD2471. Patient pathway costs for gestational diabetes, 
hypertensive disease in pregnancy and both conditions are presented in electronic supplementary Tables S2-S4, and represent the estimated costs of the patient pathway experienced by health service users for each health state.

\subsubsection{Antenatal Care and Related Costs}

Unit costs for each service or medication as outlined in the patient pathways were calculated using publicly available data on direct healthcare costs from an Australian public health perspective, and are presented in electronic supplementary Table S5. The Australian Government provides financial assistance to Australian citizens to offset the costs of medical services. These services are listed in the Medicare Benefits Schedule (MBS), and MBS data were used in this study to estimate the cost of consultations, sonography and pathology [32]. The Pharmaceutical Benefits Scheme (PBS) [33] is a government-funded scheme providing medicines at a subsidised price, and data were used to cost medications. Costs were recorded for specialist services (MBS items: 110, 116, 16404), dieticians (MBS items: 10954, 81105, 10951, 88105), midwives (MBS item: 82105), pathology (MBS items: 66542, 66500, 65070, 66509, 66512, 65129), sonography (MBS items: 55721, 16514) and medications (PBS items: 1761Q, 1629R). Unit costs for antenatal hospital admission were estimated using published data [7] and clinical expertise as described above.

\subsubsection{Delivery and Related Costs}

Diagnosis-related group (DRG) data from 2015/2016 were used for delivery costs [34] as the DRG classifies patient data from inpatient stay from admission to discharge, by diagnosis, in order to encourage containment of hospitalrelated costs. Data were adjusted to 2019 prices using the health price index [35]. Five vaginal birth outcomes (DRG codes: O02A, O02B, O60A, O60B, O60C) and three caesarean section birthing outcomes (DRG codes: O01A, $\mathrm{O} 01 \mathrm{~B}, \mathrm{O} 01 \mathrm{C}$ ) were weighted by the number of separations per code, to derive average costs for vaginal and caesarean section deliveries. Vaginal deliveries were estimated to be AUD6015, and caesarean section deliveries were estimated to be AUD11,816. Induction costs were estimated to be a proportional increase from the cost of an uncomplicated vaginal birth, and half of induced births were estimated to also have an epidural [36]. Inductions with and without an epidural were estimated as 1.54 and 1.21 times that of an uncomplicated vaginal birth [36]. Using this methodology, induction with vaginal birth was estimated as AUD8121, and induction costs separately were estimated at AUD2106.

\subsection{Subgroup Analyses}

Subgroup analysis was conducted by maternal BMI. BMI is derived by dividing weight in kilograms $(\mathrm{kg})$ by height in metres squared. Height and weight were recorded at pregnancy booking, and BMI was calculated. Booking weight is regularly used as a proxy for pre-pregnancy weight in the absence of reliable pre-pregnancy measures. Subgroup analyses were conducted using the following BMI classifications: up to $25 \mathrm{~kg} / \mathrm{m}^{2}, 25$ to up to $30 \mathrm{~kg} / \mathrm{m}^{2}$, and $30 \mathrm{~kg} / \mathrm{m}^{2}$ plus, with a further analysis for BMI of $25 \mathrm{~kg} / \mathrm{m}^{2}$ or over. Percentages of gestational diabetes, hypertensive disease in pregnancy, and both gestational diabetes and hypertensive disease cases in pregnancy by BMI category from the Monash Health dataset are presented in electronic supplementary Table S6. Higher BMI groups had higher rates of gestational diabetes and hypertensive disease in pregnancy. Of note, no differences were found in the effects of lifestyle intervention on outcomes between BMI groups in the iWIP meta-analysis [14].

\subsection{Statistical Analysis}

Analysis of Monash Health data was conducted using STATA version 15.0 (StataCorp LLC, College Station, TX, USA) to determine proportions of each health state (gestational diabetes, hypertensive disease in pregnancy, both, none of the above), and the proportions of delivery type for each health state. Decision tree models were developed in Microsoft Excel (Microsoft Corporation, Redmond, WA, USA). Data were entered into the models and the ICER for the base-case (full sample, all interventions) was calculated, representing expenditure per case (gestational diabetes, hypertensive disease in pregnancy, or both) prevented. ICERs were subsequently calculated for all subgroup and scenario analyses.

\subsection{Sensitivity Analyses}

\subsubsection{Deterministic Sensitivity Analysis}

One-way sensitivity analysis was performed for effect and cost inputs, and presented in a Tornado diagram. This type of sensitivity analysis shows the degree to which the basecase ICER is sensitive to changes in the parameters of specific independent variables while holding all other variables constant, thus enabling an assessment of the impact of uncertainty surrounding important input parameters.

\subsubsection{Scenario Analysis}

A scenario analysis was developed to investigate the use of the risk ratio for gestational diabetes for cases with 
both gestational diabetes and hypertensive disease in pregnancy. In the base-case model, the risk ratio for the health state of both gestational diabetes and hypertensive disease in pregnancy was set as the same as the risk ratio for gestational diabetes (risk ratio $=0.784$ ). In the current scenario, the risk ratio for the health state of 'both' was set to that of hypertensive disease in pregnancy (risk ratio $=0.861)$. We took a conservative estimate of the risk ratio as this would most likely be higher if an individual has two conditions concomitantly (Table 2).

\subsubsection{Probabilistic Sensitivity Analysis}

Probabilistic sensitivity analysis (PSA) was conducted to explore combined parameter uncertainty. A total of 10,000 iterations were run, with results presented on a cost-effectiveness plane. For effect inputs, the $95 \%$ CIs for the RRs for gestational diabetes, hypertensive disease in pregnancy, gestational diabetes and hypertensive disease in pregnancy, and caesarean section were used. For the cost inputs, where 95\% CIs were not available, upper and lower limits around point estimates varied by $\pm 30 \%$ [37]. To obtain a range of results for the PSA analysis, we ran four PSA models comparing gamma and uniform distributions for cost inputs $( \pm 30 \%)$, and normal and triangular distributions for effects.

\subsection{Ethical Approval}

Ethics approval for use of the Monash Health dataset was obtained from the Monash Health Human Research Ethics Committee (approval no. 14001Q).

\section{Results}

\subsection{Base-Case Analysis}

In the base-case analysis, $2.25 \%$ fewer women developed gestational diabetes and/or hypertensive disease in pregnancy (control: $11.78 \%$; intervention: $9.53 \%$ ) when lifestyle interventions were compared with usual care. Costs were close to neutral, with costs for the intervention group AUD33 higher than the control group (control: AUD8281; intervention: AUD8248). The ICER was AUD1470 per case prevented. Costs, effects and ICERS for the base-case analysis are displayed in Table 3.

\subsection{Subgroup Analysis by Body Mass Index Category}

Cost and effect differences and ICERs by BMI category are presented in Table 3. Models were cost saving for the BMI $\geq 30 \mathrm{~kg} / \mathrm{m}^{2}$ sample, while the $\mathrm{BMI} \geq 25 \mathrm{~kg} / \mathrm{m}^{2}$ sample was essentially cost-neutral. ICERs were below AUD5000 for the BMI categories of $<25 \mathrm{~kg} / \mathrm{m}^{2}$ and 25 to $<30 \mathrm{~kg} / \mathrm{m}^{2}$.

\subsection{Sensitivity Analysis}

\subsubsection{Deterministic Sensitivity Analysis}

The parameters that were most influential in the deterministic sensitivity analyses were the effect and cost of caesarean sections (Fig. 2). Using the upper limit of caesarean section costs, the ICER was cost saving, while the lower limit produced an ICER of AUD5428 per case prevented. For the lower limit of the RR for caesarean section, the ICER was cost saving, while the upper limit produced an ICER of AUD5482 per case prevented. Applying the lower limit of

Table 3 Base-case, subgroup by BMI categories and scenario analyses: effects, costs and ICERs

\begin{tabular}{|c|c|c|c|c|c|c|c|}
\hline & \multicolumn{3}{|c|}{ Effect (percentage of cases prevented) ${ }^{\mathrm{a}}$} & \multicolumn{3}{|c|}{ Cost (AUD) } & \multirow{2}{*}{$\begin{array}{l}\text { ICER } \\
\text { Per case prevented }\end{array}$} \\
\hline & Control & Intervention & Difference & Control & Intervention & Difference & \\
\hline Base-case & 11.78 & 9.53 & 2.25 & $\$ 8248$ & $\$ 8281$ & $\$ 33$ & $\$ 1470$ \\
\hline \multicolumn{8}{|c|}{ BMI subgroup analysis } \\
\hline Up to 25 & 8.71 & 7.00 & 1.71 & $\$ 7891$ & $\$ 7965$ & $\$ 74$ & $\$ 4314$ \\
\hline 25 up to 30 & 12.44 & 10.07 & 2.37 & $\$ 8388$ & $\$ 8411$ & $\$ 23$ & $\$ 974$ \\
\hline 30 plus & 18.87 & 15.38 & 3.49 & $\$ 9023$ & $\$ 8974$ & $-\$ 49$ & Dominant (cost saving) \\
\hline 25 plus & 15.25 & 12.39 & 2.86 & $\$ 8667$ & $\$ 8657$ & $-\$ 10$ & Dominant (cost saving) \\
\hline Scenario analysis ${ }^{\mathrm{b}}$ & 11.78 & 9.57 & 2.21 & $\$ 8248$ & $\$ 8283$ & $\$ 35$ & $\$ 1582$ \\
\hline
\end{tabular}

AUD Australian dollars, ICER incremental cost-effectiveness ratio, BMI body mass index

${ }^{a}$ For gestational diabetes and/or hypertensive disease

${ }^{\mathrm{b}}$ The risk ratio for the health states of gestational diabetes and hypertensive disease in pregnancy is set to risk ratio for hypertensive disease in pregnancy 


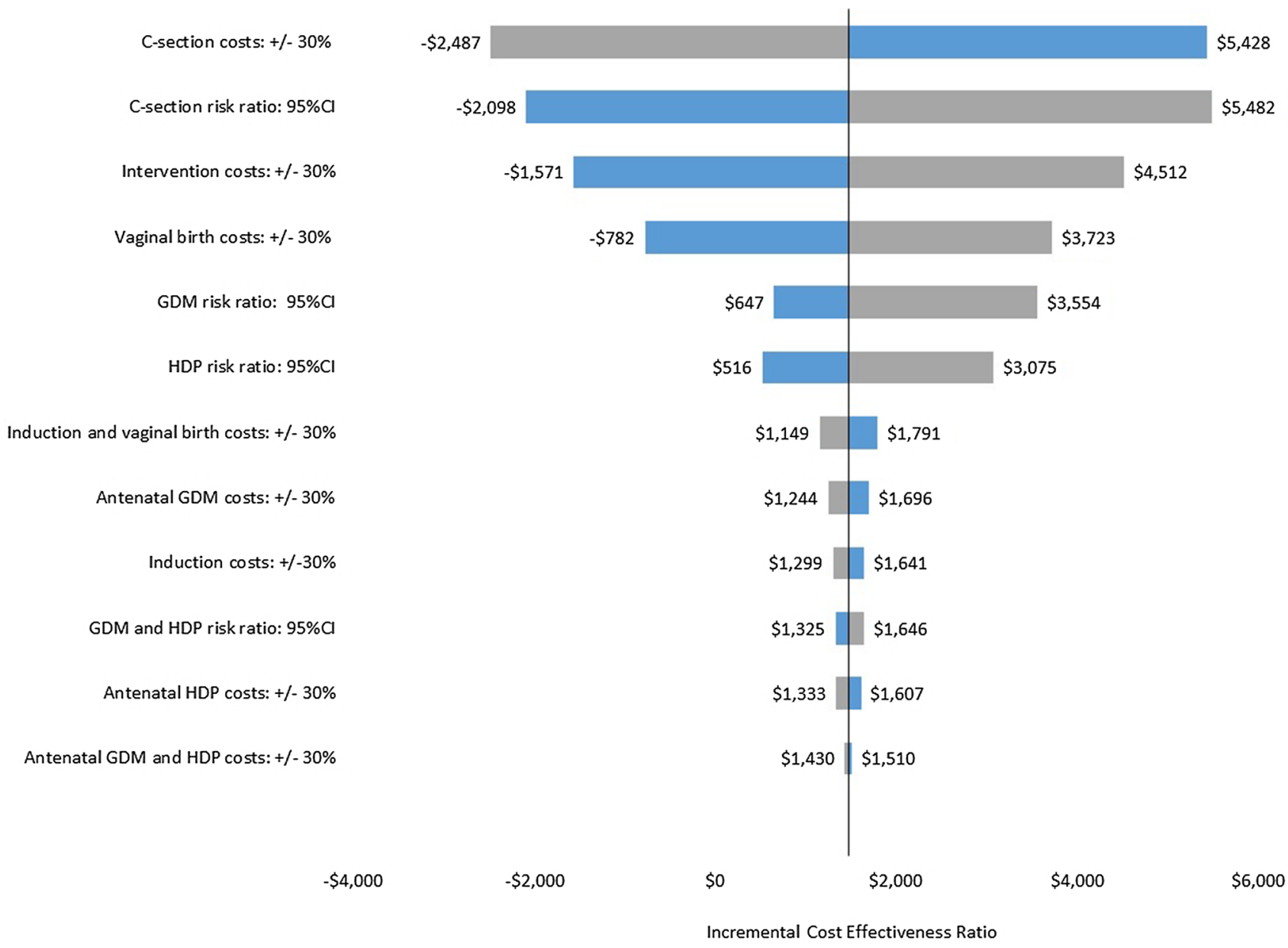

Fig. 2 Tornado chart summarising the results of deterministic sensitivity analyses, where blue bars represent the lower limit values and grey bars represent the upper limit values. GDM gestational diabetes

mellitus, HDP hypertensive disease in pregnancy, $C$-section caesarean section, $C I$ confidence interval

intervention costs, the ICER was cost saving, while applying the upper limit produced an ICER of AUD4512 per case prevented.

\subsubsection{Scenario Analysis}

When the RR for the heath state 'both' was set to the RR for hypertensive disease in pregnancy rather than the RR for gestational diabetes, the ICER was AUD1582, which was slightly higher than the base-case analysis ICER of AUD1470, as shown in Table 3.

\subsubsection{Probabilistic Sensitivity Analysis}

Probabilistic sensitivity analysis results for the base-case interventions are presented in Fig. 3. For effects, 95\% CIs were used and cost inputs were $\pm 30 \%$ from the point estimate. Results comparing different cost and effect distributions are shown in Table 4. Estimates in models using the gamma distribution for costs (and triangular or normal distributions for effects) appeared to be more cost effective than when using uniform distribution for costs (and triangular or normal distributions for effects), but had wider CIs (Table 4).

\section{Discussion}

Information on the cost effectiveness of prevention initiatives is fundamental for implementation and scale-up, but such information can be difficult to obtain and is frequently inadequate [38]. Current evidence demonstrates that excessive gestational weight gain significantly impacts pregnancy and birth outcomes for mothers and their infants [3], and that prevention interventions are effective at optimising gestational weight gain and improving lifestyle and health outcomes $[12,14]$. To inform implementation at the population level, assessing the cost effectiveness of antenatal 
Fig. 3 Cost-effectiveness plane demonstrating the probability of cost effectiveness with 10,000 iterations. The $95 \%$ uncertainty range was -AUD50,018 to AUD32,779 per case saved (probabilistic sensitivity analysis 1: gamma, normal). $A U D$ Australian dollars

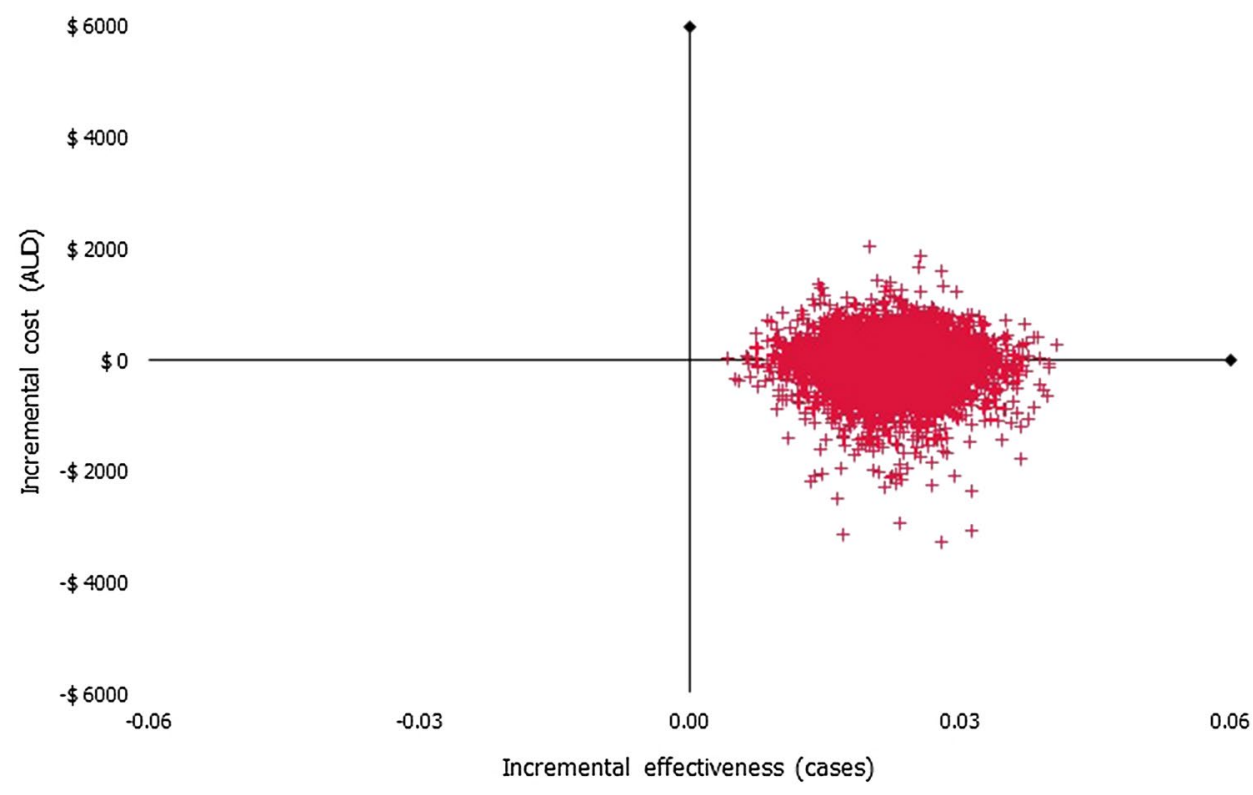

\begin{tabular}{|c|c|c|c|c|c|c|}
\hline \multirow[t]{2}{*}{ PSA analysis } & \multirow[t]{2}{*}{ Cost distribution } & \multirow[t]{2}{*}{ Effect distribution } & \multicolumn{2}{|c|}{$\begin{array}{l}\text { 95\% CI AUD for } \\
\text { ICERs }\end{array}$} & \multirow[t]{2}{*}{$\begin{array}{l}\text { North-east } \\
\text { quadrant (\%) }\end{array}$} & \multirow[t]{2}{*}{$\begin{array}{l}\text { South-east } \\
\text { quadrant (\%) }\end{array}$} \\
\hline & & & Lower & Upper & & \\
\hline 1 & Gamma & Normal & $-50,018$ & 32,779 & 44.8 & 52.2 \\
\hline 2 & Gamma & Triangular & $-44,144$ & 33,964 & 44.1 & 55.9 \\
\hline 3 & Uniform & Normal & -7789 & 10,856 & 68.9 & 31.1 \\
\hline 4 & Uniform & Triangular & -5776 & 9672 & 70.5 & 29.5 \\
\hline
\end{tabular}

PSA probabilistic sensitivity analysis, $C I$ confidence interval, AUD Australian dollars, ICERs incremental cost-effectiveness ratios
Table 4 Probabilistic sensitivity analysis results with alternative cost and effect distributions analysis was close to cost-neutral and therefore likely to be cost effective. Given the base model did not consider the substantive longer-term health outcomes of excess gestational weight gain for women (including the development of obesity, type II diabetes and heart disease) and the impact of obesity on the future child's health [42], the inclusion of long-term outcomes is likely to strengthen these findings.

Lifestyle interventions are effective across the BMI range [14]; however, adverse health outcomes are exacerbated by baseline maternal BMI, with mothers with higher weight having higher absolute risk of excess gestational weight gain, gestational diabetes, pre-eclampsia and interventional deliveries [2]. Our study has shown cost savings when baseline BMI is higher than $25 \mathrm{~kg} / \mathrm{m}^{2}$. Nonetheless, given our obesogenic environment [43], evidence that approximately $45 \%$ of pregnant women across all weight categories have excess gestational weight gain [2] and the risk of entering subsequent pregnancies at higher weight, all women are at risk of adverse health outcomes that could be prevented through improved lifestyle. Targeting women of higher weight for lifestyle interventions may also cause weight 
stigmatisation [44]. It is important that all women have access to evidence-based and effective antenatal lifestyle interventions, to optimise health in pregnancy, reduce the risk of post-partum weight retention, and reduce long-term maternal and child health complications.

Findings in the current study, utilising data from a metaanalysis with over 100 antenatal lifestyle intervention studies, were dissimilar to the results of identified cost-effectiveness studies conducted on individual randomised controlled trials [45-49]. In our systematic review, only one study was found to be cost effective (cost saving) [45], with the identified studies having relatively small samples and inadequate power to show health benefits beyond gestational weight gain. Likewise, our current results differ to that of a previous cost-effectiveness modelling study based on an earlier metaanalysis of only 36 studies [12]. Outcomes of the probabilistic sensitivity analysis in this prior cost-effectiveness study suggested that there were no significant differences between intervention and usual care groups for either intervention efficacy or costs. In contrast, with the increased number of studies included in the meta-analysis results used in the current study (where efficacy on both gestational weight gain and adverse health outcomes were demonstrated), our costeffectiveness analysis found that antenatal lifestyle interventions were likely to be cost effective, and, for higher-weight women, these interventions appeared to be cost saving.

The behavioural lifestyle intervention used to measure cost effectiveness in the current study (HeLP-her) has been delivered extensively in reproductive-age women across a series of randomised controlled trials in a range of settings and populations with consistent efficacy and high adherence rates $[24,50]$. In pregnancy, the intervention involved four face-to-face sessions of 30 min' duration integrated alongside routine antenatal care and delivered by a health coach. It is a relatively low-cost programme compared with interventions from other individual cost-effectiveness studies [16]. In the antenatal randomised controlled trial by Harrison et al. [24], this lifestyle intervention was successful in reducing excessive gestational weigh gain in a population at high risk of gestational diabetes by $1.5 \mathrm{~kg}$, compared with an average of $1.1 \mathrm{~kg}$ in the iWIP meta-analysis for all interventions [14]. As health benefits in the current study were modelled on the iWIP, where results were generated from a more limited weight difference, we may have underestimated the cost effectiveness of the HeLP-her intervention. Overall, our results highlight the need for optimally effective interventions at low cost.

\subsection{Strengths and Limitations}

The strengths of this study were the substantial meta-analysis supplying information on lifestyle intervention effectiveness, the large tertiary hospital sample that enabled a detailed understanding of proportions of health states and proportions of delivery type by health state, and the detailed costings that were developed to understand patient pathways. It is important to note that the costing model for patient pathways used in this paper is based on an estimation of the costs involved in managing and treating cases of gestational diabetes and hypertensive diseases in pregnancy. Furthermore, while we have used standard methods to cost services through the Medicare Benefit Schedule, the way these services are charged at a state and federal government level varies. This approach therefore represents an approximation of the costs involved. Variation in costs for patient pathways had little effect on the model, as noted in the sensitivity analysis.

A further limitation was that we were unable to determine the optimal intervention components and delivery modes to inform cost-effectiveness modelling, emphasising the need for more research in this area. Since the publication of the iWIP meta-analysis (used to model intervention efficacy in this study), new trials have been published, including those applying lower cost, e-health delivery methods that may have similar efficacy as face-to-face interventions. For instance, a recent effective antenatal lifestyle intervention compared electronic health delivery with a more intensive and costly face-to-face delivery and found similar efficacy for the two delivery styles [51]. This result indicated that there were opportunities for greater cost-effectiveness gains in the future. Investigation of the cost effectiveness of eHealth interventions and the inclusion of more recent studies in an updated meta-analysis would be of assistance to future research in this area.

Importantly, while the current study is the most comprehensive cost-effectiveness analysis of antenatal lifestyle interventions to date, the study only used the time horizon of early to late pregnancy. The significant benefits of prevention of obesity, type II diabetes and other maternal health benefits were not captured in this model. Given the relationship between excessive gestational weight gain and epigenetic and long-term health impacts [42], the benefits of antenatal lifestyle interventions over the longer term are likely to be important but have not been considered in this current study. Future cost-effectiveness studies should include maternal and child health outcomes over a longer time horizon, and could also investigate outcome measures such as QALYs and productivity loss over the longer term.

\section{Conclusions}

In the most comprehensive cost-effectiveness analysis of antenatal lifestyle interventions to date, we suggest that lifestyle interventions are cost effective for reducing adverse maternal outcomes in pregnancy, and that interventions for 
mothers in higher weight categories are cost saving. These findings are novel as they advance prior knowledge based on smaller samples that failed to show cost effectiveness. To effectively inform guidelines and scale-up interventions into policy and practice, future research is needed to establish the characteristics of efficacious interventions that can be delivered at low cost. Cost-effectiveness modelling incorporating the longer-term health benefits of antenatal lifestyle interventions is also required. This work supports guideline and policy recommendations to implement population-wide antenatal lifestyle interventions.

Acknowledgements The authors would like to acknowledge Professor Danny Liew, Dr Catherine Keating and the Monash Outcome Research and Health Economics (MORE) team for their support in completing this project. The model used in this study was provided to the journal's peer reviewers for their reference when reviewing the manuscript.

Author Contributions CB, ZA, HT, CH and ST conceived the study. $\mathrm{CB}, \mathrm{ZA}, \mathrm{JB}$ and $\mathrm{RB}$ developed the costings for the patient pathways. $\mathrm{CH}$ and HT led the HeLP-her intervention and contributed data on the intervention costs; HT led the formation and analysis of the Monash Health dataset on outcomes; ST led the iWIP individual patient data meta-analysis, with HT and $\mathrm{CH}$ named as collaborators; and $\mathrm{CB}$, HS, $\mathrm{HT}, \mathrm{ST}, \mathrm{BH}$ and ZA conceived the cost-effectiveness questions. CB and ZA completed the cost-effectiveness modelling, CB wrote the initial draft, and all authors had input into drafting and critical review of the manuscript. The corresponding author attests that all listed authors meet the authorship criteria and that no others meeting the criteria have been omitted. The first (CB) and last (ZA) authors affirm that the manuscript is an honest, accurate and transparent account of the study being reported; that no important aspects of the study have been omitted; and that any discrepancies from the study as planned (and, if relevant, registered) have been explained.

\section{Compliance with Ethical Standards}

Funding Funding for this research was provided from the Australian Government's Medical Research Future Fund (Through The Australian Prevention Partnership Centre) (MRFF), which provides funding to support health and medical research and innovation with the objective of improving the health and wellbeing of Australians. MRFF funding has been provided to The Australian Prevention Partnership Centre under the MRFF Boosting Preventive Health Research Program. Further information on the MRFF is available at www.health.gov.au/mrff. Individual funding: The International Diabetes Federation and the Jack Brockhoff Foundation supported the HeLP-her study. The UK National Institute of Health Research supported the iWIP research. The Australian National Health and Medical Research Council (NHMRC) supports fellowships for HT, JB and BH. CB was supported by an Australian Government Research Training Program (RTP) Scholarship.

Conflict of interest Cate Bailey, Helen Skouteris, Cheryce L. Harrison, Jacqueline Boyle, Rebeccah Bartlett, Briony Hill, Shakila Thangaratinam, Helena Teede, and Zanfina Ademi declare they have no conflicts of interest.

Ethics No ethics approval was required.

Data Availability Statement Data are available in published form for ORs [14] and costing data are presented in the electronic supplemen- tary files. The Monash Health dataset is not publically available due to third-party restrictions. The model is available upon reasonable request to the authors.

Related Manuscript No authors have a related or duplicate manuscript under consideration or accepted for publication elsewhere.

Open Access This article is licensed under a Creative Commons Attribution-NonCommercial 4.0 International License, which permits any non-commercial use, sharing, adaptation, distribution and reproduction in any medium or format, as long as you give appropriate credit to the original author(s) and the source, provide a link to the Creative Commons licence, and indicate if changes were made. The images or other third party material in this article are included in the article's Creative Commons licence, unless indicated otherwise in a credit line to the material. If material is not included in the article's Creative Commons licence and your intended use is not permitted by statutory regulation or exceeds the permitted use, you will need to obtain permission directly from the copyright holder.To view a copy of this licence, visit http://creativecommons.org/licenses/by-nc/4.0/.

\section{References}

1. World Health Organization. Health topics: obesity. 2018. http:// www.who.int/topics/obesity/en/. Accessed 20 Feb 2018.

2. Cheney K, Farber R, Barratt AL, et al. Population attributable fractions of perinatal outcomes for nulliparous women associated with overweight and obesity, 1990-2014. Med J Aust. 2018;208:119-25. https://doi.org/10.5694/mja17.00344.

3. Goldstein RF, Abell SK, Ranasinha S, et al. Association of gestational weight gain with maternal and infant outcomes: a systematic review and meta-analysis. JAMA. 2017;317:2207-25. https ://doi.org/10.1001/jama.2017.3635.

4. Institute of Medicine and National Research Council Committee to Reexamine IOM Pregnancy Weight Guidelines. Re-examining IOM Pregnancy Weight Guidelines. In: Rasmussen KM, Yaktine AL, editors. Weight gain during pregnancy: reexamining the guidelines. Washington, DC: National Academies Press; 2009.

5. Meregaglia M, Dainelli L, Banks H, et al. The short-term economic burden of gestational diabetes mellitus in Italy. BMC Pregnancy Childbirth. 2018;18(1):58.

6. Anderberg E, Carlsson KS, Berntorp K. Use of healthcare resources after gestational diabetes mellitus: a longitudinal casecontrol analysis. Scand J Public Health. 2012;40:385-90. https:// doi.org/10.1177/1403494812449923.

7. Fox A, McHugh S, Browne J, et al. Estimating the cost of preeclampsia in the healthcare system. Hypertension. 2017;70:1243-9. https://doi.org/10.1161/hypertensionaha.117.09499.

8. Xu T, Dainelli L, Yu K, et al. The short-term health and economic burden of gestational diabetes mellitus in China: a modelling study. BMJ Open. 2017;7:1-9. https://doi.org/10.1136/bmjop en-2017-018893.

9. Australian Institute of Health and Welfare. Incidence of gestational diabetes in Australia. Canberra: Australian Institute of Health and Welfare; 2019.

10. Dall TM, Yang W, Halder P, et al. The economic burden of elevated blood glucose levels in 2012: diagnosed and undiagnosed diabetes, gestational diabetes mellitus, and prediabetes. Diabetes Care. 2014;37:3172-9. https://doi.org/10.2337/dc14-1036.

11. Stevens W, Shih T, Incerti D, et al. Short-term costs of preeclampsia to the United States health care system. Am J Obstet 
Gynecol. 2017;217(237-248):e16. https://doi.org/10.1016/j. ajog.2017.04.032.

12. Rogozińska E, Marlin N, Jackson L, et al. Effects of antenatal diet and physical activity on maternal and fetal outcomes: individual patient data meta-analysis and health economic evaluation. Health Technol Assess. 2017;21(41):1-158. https://doi.org/10.3310/ hta21410.

13. Walker R, Bennett C, Blumfield M, et al. Attenuating pregnancy weight gain - what works and why: a systematic review and metaanalysis. Nutrients. 2018;10:4-6. https://doi.org/10.3390/nu100 70944.

14. The International Weight Management in Pregnancy Collaborative Group. Effect of diet and physical activity based interventions in pregnancy on gestational weight gain and pregnancy outcomes: meta-analysis of individual participant data from randomised trials. BMJ. 2017;358:j3119. https://doi.org/10.1136/bmj.j3119.

15. Rogozinska E, D’Amico MI, Khan KS, et al. Development of composite outcomes for individual patient data (IPD) meta-analysis on the effects of diet and lifestyle in pregnancy: a Delphi survey. BJOG. 2015;123:190-8. https://doi.org/10.1111/1471-0528.13764.

16. Bailey C, Skouteis H, Teede H, Hill B, De Courten B, Walker R, Liew D, Thangaratinam S, Ademi Z. Are Lifestyle Interventions to Reduce Excessive Gestational Weight Gain Cost Effective? A Systematic Review. Curr Diab Rep. 2020;. https://doi.org/10.1007/ s11892-020-1288-1.

17. Abell SK, Teede HJ. The IADPSG diagnostic criteria identify women with increased risk of adverse pregnancy outcomes in Victoria. Aust N Z J Obstet Gynaecol. 2017;57:564-8. https:// doi.org/10.1111/ajo.12676.

18. Abell SK, Suen M, Pease A, et al. Pregnancy outcomes and insulin requirements in women with type 1 diabetes treated with continuous subcutaneous insulin infusion and multiple daily injections: cohort study. Diabetes Technol Ther. 2017;19:280-7. https://doi. org/10.1089/dia.2016.0412.

19. World Health Organization. Health topics: body mass indexBMI. 2019. http://www.euro.who.int/en/health-topics/diseaseprevention/nutrition/a-healthy-lifestyle/body-mass-index-bmi. Accessed 25 Mar 2019.

20. Nankervis A, McIntyre HD, Moses R, et al. ADIPS Consensus Guidelines for the Testing and Diagnosis of Gestational Diabetes Mellitus in Australia. 2014. http://www.adips.org/downloads/ ADIPSConsensusGuidelinesGDM-03.05.13VersionACCEPT EDFINAL.pdf.

21. Crowther CA, Hiller JE, Moss JR, McPhee AJ, Jeffries WS, Robinson JS. Effect of treatment of gestational diabetes mellitus on pregnancy outcomes. N Engl J Med. 2005;352:2477-86.

22. Grant RL. Converting an odds ratio to a range of plausible relative risks for better communication of research findings. BMJ. 2014;348:1-7. https://doi.org/10.1136/bmj.f7450.

23. Viera AJ. Odds ratios and risk ratios: what's the difference and why does it matter? South Med Assoc. 2008;101:730-4.

24. Harrison CL, Lombard CB, Teede HJ. Limiting postpartum weight retention through early antenatal intervention: the HeLP-her randomised controlled trial. Int J Behav Nutr Phys Act. 2014;11:1-8. https://doi.org/10.1186/s12966-014-0134-8.

25. Harrison C, Lombard CB, Strauss BJ, Teede HJ. Optimizing healthy gestational weight gain in women at high risk of gestational diabetes: a randomized controlled trial. Obesity. 2013;21(5):904-9.

26. Teede H, Harrison CL, Teh WT, et al. Gestational diabetes: development of an early risk prediction tool to facilitate opportunities for prevention. Aust N Z J Obstet Gynaecol. 2011;6:499-504.

27. Health Monash. Hypertensive disorders in pregnancy pre eclampsia/eclampsia. Clinical Guideline. Melbourne: Monash Health; 2017.
28. Health Monash. Diabetes-intrapartum and intial postnatal management: clinical guidelines. Melbourne: Monash Health; 2014.

29. Health Monash. Fetal surveillance in pregnancy for women with diabetes. Melbourne: Monash Health; 2016.

30. The Royal Women's Hospital. Diabetes Mellitus: management of gestational diabetes. Melbourne: The Royal Women's Hospital; 2019.

31. Australian Institute of Health and Welfare. Diabetes in pregnancy: its impact on Australian women and their babies. Diabetes series no. 14. Cat. no. CVD 52. Canberra, ACT: Australian Institute of Health and Welfare; 2010.

32. Department of Health. MBS Online: Medicare Benefits Schedule. 2018. http://www.mbsonline.gov.au/internet/mbsonline/publi shing.nsf/Content/Downloads-201811. Accessed 18 Oct 2018.

33. Department of Health. Schedule of Pharmaceutical Benefits. Canberra: Department of Health; 2019.

34. Independent Hospital Pricing Authority. National Hospital Cost Data Collection, Public Hospitals Cost Report, Round 20 (Financial year 2015-16). Darlinghurst: Independent Hospital Pricing Authority; 2018.

35. Australian Institute of Health and Welfare. Health Expenditure Australia 2016-17. Canberra: Australian Institute of Health and Welfare; 2018.

36. Tracy SK, Tracy MB. Costing the cascade: estimating the cost of increased obstetric intervention in childbirth using population data. BJOG. 2003;110:717-24. https://doi.org/10.111 1/j.1471-0528.2003.02045.x.

37. Blank PR, Ademi Z, Lu X, et al. Herpes zoster vaccine: a health economic evaluation for Switzerland. Hum Vaccines Immunother. 2017;13:1495-504. https://doi.org/10.1080/21645515.2017.13089 87.

38. Jakob Z, Gurria A. Promoting health, preventing disease: the economic case. Forward. New York: Open University Press; 2015.

39. Paris V, Belloni A. Value in pharmaceutical pricing. OECD Health Work Pap. 2013;63:1-74. https://doi.org/10.1787/5k43jc9v6k nx-en.

40. Edney LC, Haji A, Afzali H, Cheng TC, Karnon J. Estimating the reference incremental cost-effectiveness ratio for the Australian health system. Pharmacoeconomics. 2018;36:239-52. https://doi. org/10.1007/s40273-017-0585-2.

41. Appleby J, Devlin N, Parkin D. NICE's cost effectiveness threshold. BMJ. 2007;335:358-9.

42. Langley-Evans SC. Nutrition in early life and the programming of adult disease: a review. J Hum Nutr Diet. 2015;28:1-14. https ://doi.org/10.1111/jhn.12212.

43. Swinburn B, Kraak V, Rutter H, et al. Strengthening of accountability systems to create healthy food environments and reduce global obesity. Lancet. 2015;385:2534-45. https://doi. org/10.1016/S0140-6736(14)61747-5.

44. Major B, Hunger JM, Bunyan DP, Miller CT. The ironic effects of weight stigma. J Exp Soc Psychol. 2014;51:74-80. https://doi. org/10.1016/j.jesp.2013.11.009.

45. Broekhuizen K, Simmons D, Devlieger R, et al. Cost-effectiveness of healthy eating and/or physical activity promotion in pregnant women at increased risk of gestational diabetes mellitus: economic evaluation alongside the DALI study, a European multicenter randomized controlled trial. Int J Behav Nutr Phys Act. 2018;15:1-12. https://doi.org/10.1186/s12966-018-0643-y.

46. Oostdam N, Bosmans J, Woulters MGAJ, et al. Cost-effectiveness of an exercise program during pregnancy to prevent gestational diabetes: results of an economic evaluation alongside a randomised controlled trial. BMC Pregnancy Childbirth. 2012;12:64.

47. Dodd JM, Ahmed S, Karnon J, et al. The cost-effectiveness of providing antenatal lifestyle advice for women who are overweight or obese: the LIMIT randomised trial. BMC Obes. 2015;2:1-9. https://doi.org/10.1186/s40608-015-0046-4. 
48. Poston L, Bell R, Briley AL, et al. Improving pregnancy outcome in obese women: the UK Pregnancies Better Eating and Activity randomised controlled Trial. Program Grants Appl Res. 2017;5:1-414. https://doi.org/10.3310/pgfar05100.

49. Kolu P, Raitanen J, Rissanen P, Luoto R. Cost-effectiveness of lifestyle counselling as primary prevention of gestational diabetes mellitus: findings from a cluster-randomised trial. PLoS One. 2013;8(2):e56392. https://doi.org/10.1371/journal.pone.0056392.

50. Lombard C, Harrison C, Kozica S, et al. Preventing weight gain in women in rural communities: a cluster randomised controlled trial. PLoS Med. 2016;13(1):e1001941. https://doi.org/10.1371/ journal.pmed.1001941.

51. Redman LM, Gilmore LA, Breaux J, et al. Effectiveness of SmartMoms, a Novel eHealth Intervention for Management of Gestational Weight Gain: randomized Controlled Pilot Trial. JMIR Mhealth Uhealth. 2017;5(9):e133. https://doi.org/10.2196/mheal th.8228. 